\title{
Avaliação da solicitação de exames laboratoriais em um hospital universitário: consequências para a clínica e a gestão
}

\author{
Assessment of request of laboratory tests in a university \\ hospital: consequences for Clinical and Management
}

\author{
André Felipe Maciel Moreno Gomes ${ }^{1}$ (D), Altacílio Aparecido Nunes ${ }^{1}$
}

\begin{abstract}
Resumo
Introdução: Os princípios estabelecidos pelo SUS fizeram com que o atual cenário da saúde pública ganhasse complexidade envolvendo o financiamento e o suporte para a sustentabilidade do sistema. Há relatos sobre o excesso no uso de recursos diagnósticos, muitas vezes desnecessários, com consequências para os pacientes e o orçamento dos serviços de saúde, sobretudo hospitais. Objetivo: Avaliar os resultados qualitativos e quantitativos de exames laboratoriais solicitados nos ambulatórios de Cardiologia e Endocrinologia do Hospital das Clínicas da Faculdade de Medicina de Ribeirão Preto da Universidade de São Paulo. Método: Foi realizado um estudo transversal com análise dos exames solicitados nos ambulatórios de Endocrinologia e Cardiologia entre julho/2012 e julho/2013. Resultados: A quantidade de exames foi semelhante ao longo dos 12 meses, e a relação de exames/paciente foi maior no ambulatório de Cardiologia, com 10,36 exames, do que no ambulatório de Endocrinologia, com os 5,91 exames. Analisando os subelementos dos exames, os resultados foram próximos: em Cardiologia, a taxa média de elementos alterados foi de 20,79\%, ao passo que, em Endocrinologia, o valor médio foi de 22,39\%. Conclusão: Entender o comportamento de médicos na solicitação dos exames laboratoriais em um hospital de grande porte pode estimular os líderes e os gestores a buscar propostas para melhorar o gerenciamento desses grandes centros de alta complexidade assistencial, buscando a eficiência e a qualidade na assistência e no ensino.
\end{abstract}

Palavras-chave: exames laboratoriais; economia da saúde; gestão em saúde; hospital.

\begin{abstract}
Background: The increasing demand of medical care according to the principles established by the Brazilian Unified Health System made the current situation of public health gain complexity involving funding and support to maintain these services. There are reports of excessive use of diagnostic resources often unnecessary, with consequences for patients and for the budget of health services, especially hospitals. Objective: It was to evaluate the qualitative and quantitative results of laboratory tests ordered in the outpatient clinics of Cardiology and Endocrinology of the Hospital of the Ribeirão Preto Medical School-USP. Method: A cross-sectional study was carried out. An analysis of the exams was performed in outpatient clinics in the endocrinology and cardiology between July/2012 and July/2013. Results: The number of tests was similar over the 12 months, and the examination/patient relationship was higher in the cardiology clinic with 10.36 versus 5.91 observed in endocrinology clinic. Besides that, it was observed the sub-elements of the tests, which demonstrated similar results: in Cardiology average rate of change elements was $20.79 \%$, whereas in endocrinology, the average was $22.39 \%$. Conclusion: Understanding the behavior of physicians in requesting laboratory examinations in a large hospital can stimulate leaders and managers to seek proposals to improve the management of these large centers of high complexity of care. Thus, they can seek efficiency, quality of care and teaching.

Keywords: laboratory testing; health economics; management health; hospital.

${ }_{1}^{1}$ Programa de Mestrado Profissional em Gestão de Organizações de Saúde, Faculdade de Medicina de Ribeirão Preto, Universidade de São Paulo (USP) - Ribeirão Preto (SP), Brasil.

Trabalho realizado no Hospital das Clínicas, Faculdade de Medicina de Ribeirão Preto, Universidade de São Paulo (USP) - Ribeirão Preto (SP), Brasil. Endereço para correspondência: Altacílio Aparecido Nunes - Departamento de Medicina Social, Faculdade de Medicina de Ribeirão Preto, Universidade de São Paulo (USP), Av. Bandeirantes, 3900 - CEP: 14049-900 - Ribeirão Preto (SP), Brasil - Email: altacilio@fmrp.usp.br

Fonte de financiamento: nenhuma.

Conflito de interesses: nada a declarar.
\end{abstract}

Este é um artigo publicado em acesso aberto (Open Access) sob a licença Creative Commons Attribution, que permite uso, distribuição e reprodução em qualquer meio, sem restrições desde que o trabalho original seja corretamente citado. 


\section{INTRODUÇÃO}

A pluralização da assistência à saúde da população em todos os níveis de atenção, segundo os princípios de universalidade, equidade e integralidade, fez com que o atual cenário da saúde pública ganhasse grande complexidade envolvendo o financiamento e o suporte para a manutenção desses serviços, de acordo com o estabelecido pelo Sistema Único de Saúde (SUS). Nesse contexto, destaca-se o emprego de recursos diagnósticos, insumos e tecnologias envolvidos na atenção ao paciente, em razão dos conflitos entre aspectos econômicos e benefícios alcançados ${ }^{1,2}$.

As facilidades e o aumento do acesso à informação também permitiram aos pacientes maior aquisição de conhecimento sobre saúde, obtendo informações na internet e em outras mídias sobre medicamentos, posologias e tratamentos alternativos, além de ter feedback com outras pessoas e profissionais a respeito de procedimentos médicos. Muitas vezes, pacientes chegam ao consultório com lista de exames feitos, impondo ao médico a solicitação, criticando e julgando o comportamento desse profissional quando não conseguem ter seu pedido satisfeito ${ }^{3}$. Dessa forma, os exames diagnósticos estão totalmente ligados às políticas públicas de saúde e inseridos nelas ao representarem um importante papel na prevenção, no diagnóstico, no tratamento e no acompanhamento das enfermidades em geral, sendo preponderantes para a qualidade de vida do paciente. Esses exames diagnósticos podem ser divididos nas categorias de análises clínicas e de diagnóstico por imagem ${ }^{4,5}$.

No plano da demanda, a quantidade de exames diagnósticos pode variar de acordo com o ambiente em que são realizados os atendimentos, sendo mais elevada em hospitais em decorrência do nível de complexidade dos serviços prestados. Assim, observando a relevância dessa questão envolvendo a demanda de procedimentos diagnósticos para o sistema de saúde de uma forma geral, alguns autores vêm discutindo a real necessidade e coerência que envolvem o uso de tais recursos. Há evidências e estudos que indicam excesso no número de solicitações de exames diagnósticos, uma "superutilização" desses recursos que, muitas vezes, seguem diferentes critérios de indicação, ou seja, há uma heterogeneidade acerca da interpretação e da compreensão dos achados, ou que são solicitados por convenção antes mesmo da avaliação física, além de outros fatores individuais ${ }^{6-8}$.

Alguns fatores que parecem contribuir mais sensivelmente para o número de exames considerados desnecessários incluem as características da instituição hospitalar, sendo que os hospitais de ensino correspondem a uma parcela significativamente maior, bem como o tempo de experiência, a especialidade e a hierarquia do médico, a logística e o sistema de informação relacionado ao atendimento, o quadro clínico do paciente, os fatores comportamentais e o conhecimento sobre custos de procedimentos diagnósticos ${ }^{9-13}$.
Assim, o objetivo deste artigo é apresentar os resultados dos principais parâmetros laboratoriais de exames solicitados nas áreas de Cardiologia e Endocrinologia em um hospital universitário de alta complexidade, buscando identificar se as solicitações dos exames e o uso desses recursos de saúde ocorreram de forma racional e coerente. Pretende-se, assim, estimular novas propostas associadas à melhora na utilização dos recursos e na gestão hospitalar, bem como fomentar estudos posteriores que abordem o uso de outros recursos e tecnologias em saúde para que possam ser estendidos e aplicados ao sistema de saúde.

\section{MÉTODO}

Trata-se de um estudo transversal, com descrição e análise dos resultados dos principais parâmetros laboratoriais e de respectivos custos em exames solicitados nas áreas de Endocrinologia e Cardiologia da divisão de Clínica Médica do Hospital das Clínicas da Faculdade de Medicina de Ribeirão Preto da Universidade de São Paulo (HCFMRP-USP), realizados entre julho de 2012 e julho de 2013.

O HCFMRP-USP é um hospital de ensino, mantido pelo governo do Estado de São Paulo, vinculado administrativamente à Secretaria de Estado da Saúde e associado à Universidade de São Paulo. Está inserido em um sistema hierarquizado e referenciado de saúde, mantido com recursos tripartite, provenientes do SUS, das prefeituras municipais e do governo do Estado. Tem função assistencial, assim como de ensino e pesquisa aplicada. É um centro de referência no atendimento médico hospitalar do interior do Estado de São Paulo, abrangendo uma população de mais de 3,5 milhões de habitantes ao atender às demandas dos Departamentos Regionais de Saúde (DRS) de Ribeirão Preto, Franca, Araraquara e Barretos, além de outras regiões do Estado de São Paulo e até mesmo de outros Estados e países. No hospital, são realizados em média cerca de 300 mil exames laboratoriais por mês.

Foi realizada uma análise dos resultados de exames por meio do sistema eletrônico de gestão do hospital (HC-Athos ${ }^{\circledR}$ ), solicitados em regime de primeira consulta e retorno, nas áreas de Endocrinologia (ambulatórios ENA [Endocrinologia de adultos], END [Endocrinologia - Diabetes], ETP [Endocrinologia - Tireoide e Paratireoide]) e Cardiologia (CAA) durante o período de julho/2012 a julho/2013. A escolha dessas áreas clínicas se justifica pelo fato de ambas terem grande número de protocolos validados para diversas doenças, facilitando o desenvolvimento do estudo, e ainda por causa de sua relevância e representatividade epidemiológica. A opção pela análise desses parâmetros laboratoriais ocorreu por serem os mais recorrentes e relevantes nesses ambulatórios e por estarem associados ao diagnóstico de doenças com alta importância e prevalência, como distúrbios cardiovasculares, incluindo hipertensão, 
doenças hormonais relacionadas à tireoide e demais glândulas, diabetes etc.

Para a definição do tamanho amostral (quantidade de exames analisados), os seguintes dados foram empregados: população-alvo de $36 \mathrm{mil}$ (total de consultas realizadas em 2012 em Cardiologia e Endocrinologia), poder estatístico de $80 \%$, prevalência de exames alterados igual a $40 \%$ (com base em dados na literatura), erro amostral absoluto tolerado de $3 \%$ e nível de significância de 5\%. Assim, o tamanho amostral resultante foi de 997 consultas. No entanto, realizou-se a análise de 3.721 exames de pacientes por meio do sistema HC-Athos ${ }^{\circledR}$. Para a apresentação dos resultados, utilizou-se da estatística descritiva com cálculo de número absoluto e percentual, medida de tendência central (média e mediana) e medidas de dispersão, enquanto, na análise comparativa de diferenças entre as variáveis, empregou-se o teste $\mathrm{Z}$ para proporções. $\mathrm{Na}$ comparação de médias, foi empregado o teste $t$ de Student para amostras independentes.

Considerando-se que, na presente pesquisa, utilizou-se apenas de informações obtidas em banco de dados, de maneira agrupada, sem identificação de sujeitos ou entrevistas e/ou procedimentos com pacientes, nem consultas a prontuários, não houve necessidade de apreciação pelo Comitê de Ética em Pesquisa.

\section{RESULTADOS}

No Gráfico 1, é apresentado o número absoluto de exames realizados em cada um dos meses estudados nos ambulatórios de Cardiologia e Endocrinologia.

Pode-se observar que as demandas em número absoluto de exames nos dois ambulatórios foram diferentes ao longo do ano, sendo maior no ambulatório de Endocrinologia. Ao se comparar a média anual de solicitação de exames, na Cardiologia foram encontrados 870 exames, e na Endocrinologia, 1.338, resultando em diferença estatisticamente significativa $(\mathrm{p}<0,05)$. Considerando-se a comparação do segundo semestre de 2012 com o primeiro semestre de 2013, a média do número de exames realizados na Endocrinologia foi de 1.350 e 1.325, respectivamente, enquanto na Cardiologia foi de 942 e 797, respectivamente, sendo que em ambos os casos não foi detectada diferença significativa $(\mathrm{p}>0,05)$.

Na Tabela 1, podem ser observados os números absolutos de pacientes, exames e consultas realizados pelos ambulatórios de Cardiologia e Endocrinologia.

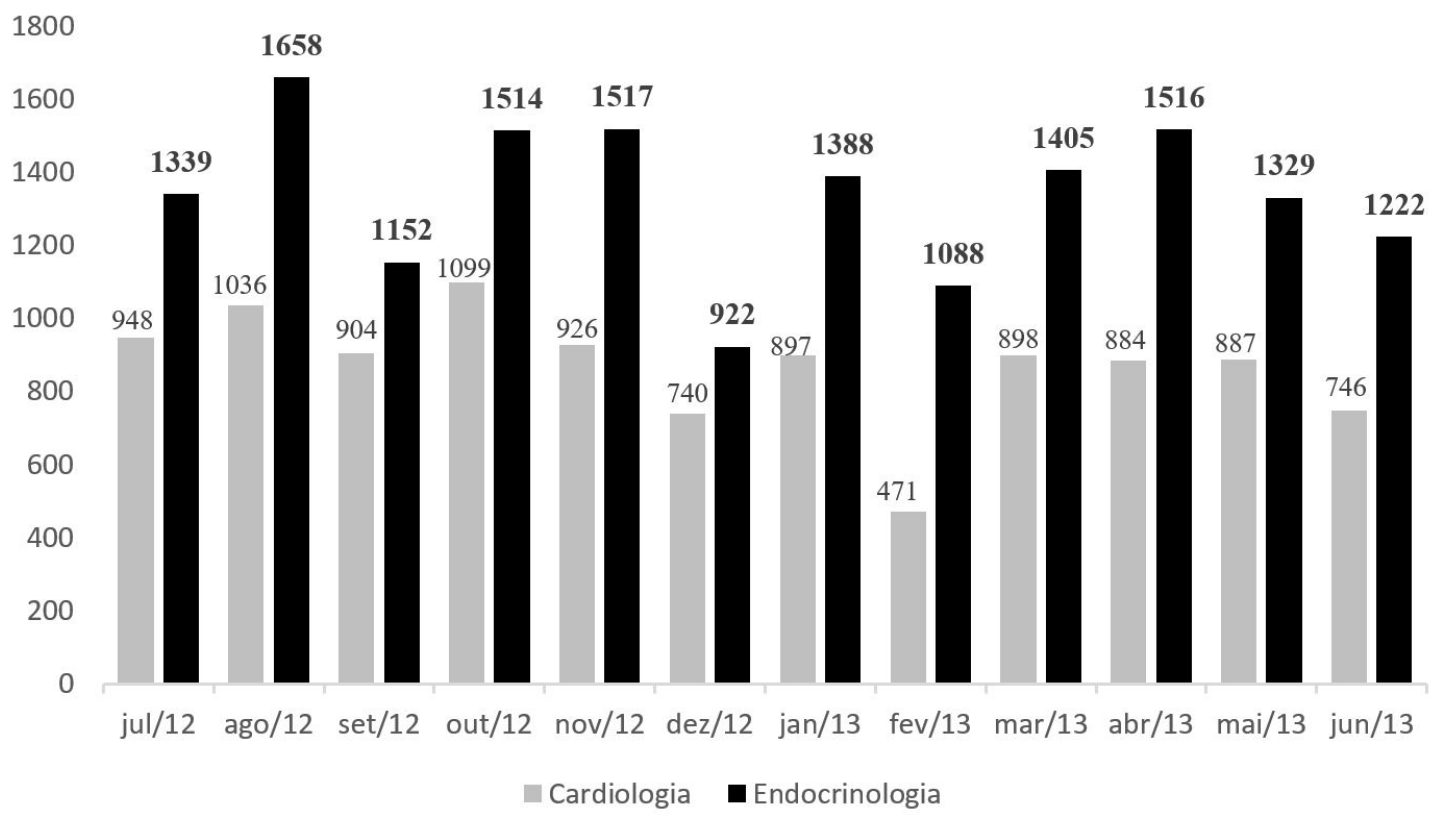

Gráfico 1. Quantidades de exames em Cardiologia e Endocrinologia na Clínica Médica do Hospital das Clínicas da Faculdade de Medicina de Ribeirão Preto da Universidade de São Paulo (HCFMRP-USP), no período entre julho de 2012 e julho de 2013. Média anual: Endocrinologia = 870; Cardiologia $=1.338 ; p<0,05$

Tabela 1. Resumo das solicitações de exames realizados nos ambulatórios de Cardiologia e Endocrinologia do Hospital das Clínicas da Faculdade de Medicina de Ribeirão Preto da Universidade de São Paulo (HCFMRP-USP), no período entre julho de 2012 e julho de 2013

\begin{tabular}{lccccccc}
\multicolumn{1}{c}{ Ambulatório } & Pacientes & Exames & Consultas & $\begin{array}{c}\text { Exames/ } \\
\text { paciente }\end{array}$ & Valor de p & $\begin{array}{l}\text { Exames/ } \\
\text { consulta }\end{array}$ \\
Cardiologia & 1007 & 10436 & 5753 & 10,36 & & 1,81 & 1,29 \\
Endocrinologia & 2714 & 16052 & 12464 & 5,91 & 0,033 & 0,043 \\
Total & 3721 & 26488 & 18217 & & & & \\
\hline
\end{tabular}


Como já demonstrado no Gráfico 1 e detalhado na Tabela 1, a quantidade absoluta e proporcional de exames realizados no ambulatório de Endocrinologia foi maior do que no de Cardiologia, o que se explica pelo fato de o referido ambulatório ter o maior número de pacientes. Na Cardiologia, a relação de exames por paciente se mostrou quase que o dobro do observado na Endocrinologia com diferença significativa (diferença de taxa $=4,45$ [IC95\%: 4,25-4,64]; p < 0,05), o que sugere que tais diferenças podem ser explicadas pelas particularidades das doenças atendidas em cada uma das duas especialidades. Ao se comparar o número de exames por consulta, nota-se que na Cardiologia foi de 1,81, ao passo que na Endocrinologia foi de 1,29, encontrando-se diferença significativa (diferença de taxas $=0,52$ [IC95\%: 0,48-0,56]; $\mathrm{p}<0,05$ ).

Nas Tabelas 2 e 3, são mostrados os resultados com indicadores de anormalidade para cada um dos parâmetros laboratoriais em ambos os ambulatórios, sendo possível observar o comportamento de cada elemento e facilitando a compreensão no contexto apresentado. Comparando-se a Cardiologia com a Endocrinologia ENA e a Endocrinologia END + ETP (Tabela 2), nota-se que, nos exames em que foram encontradas diferenças estatisticamente significativas ( $p<0,05)$, em regra, estas representavam um maior percentual de exames anormais no ambulatório de Cardiologia, denotando as características da população e das doenças atendidas nessa especialidade.

Pode ser observado que em Cardiologia a taxa média de elementos alterados foi de apenas $20,79 \%$, e, comparando as proporções com os valores considerados normais, encontraram-se diferenças estatisticamente significativas em todos os parâmetros $(\mathrm{p}<0,05)$. Com exceção de alguns elementos que destoaram do valor médio, esses resultados podem levar à discussão sobre a necessidade de realização dos exames relacionados, tendo em consideração o perfil dos pacientes e as doenças associadas.

Ao se analisar os resultados dos exames solicitados nos ambulatórios (ENA e END + ETP) de Endocrinologia (Tabela 3), visualiza-se o comportamento dos elementos em cada um dos

Tabela 2. Análise comparativa dos elementos de exames de bioquímica e hemograma realizados nos ambulatórios de Cardiologia e Endocrinologia do Hospital das Clínicas da Faculdade de Medicina de Ribeirão Preto da Universidade de São Paulo (HCFMRP-USP), no período entre julho de 2012 e julho de 2013

\begin{tabular}{|c|c|c|c|c|c|}
\hline \multicolumn{4}{|c|}{ Elementos alterados dos exames (\%) } & \multirow{2}{*}{$\begin{array}{c}\text { Valor de } \mathbf{p} \\
\text { (Cardiologia } v s \\
\text { Endocrinologia } \\
\text { ENA) }\end{array}$} & \multirow{2}{*}{$\begin{array}{c}\text { Valor de p } \\
\text { (Cardiologia } v s \\
\text { Endocrinologia } \\
\text { END + ETP) }\end{array}$} \\
\hline Parâmetro & Cardiologia & $\begin{array}{c}\text { Endocrinologia } \\
\text { ENA }\end{array}$ & $\begin{array}{l}\text { Endocrinologia } \\
\text { END + ETP }\end{array}$ & & \\
\hline Creatinina & 29,22 & - & - & NA & NA \\
\hline Ureia & 36,15 & - & - & NA & NA \\
\hline Sódio & 14,09 & 13,52 & 14,24 & & \\
\hline CKMB & 30,28 & - & - & NA & NA \\
\hline Cálcio iônico & 28,91 & 37,23 & 24,57 & 0,041 & 0,045 \\
\hline Potássio & 19,62 & 19,32 & 20,63 & 0,041 & 0,071 \\
\hline \multicolumn{4}{|c|}{ Hemograma } & & \\
\hline Parâmetro & Cardiologia & $\begin{array}{c}\text { Endocrinologia } \\
\text { ENA }\end{array}$ & $\begin{array}{c}\text { Endocrinologia } \\
\text { END + ETP }\end{array}$ & & \\
\hline Glóbulos vermelhos & 37,77 & 28,02 & 36,63 & 0,032 & 0,061 \\
\hline Glóbulos brancos & 13,14 & 14,22 & 14,72 & 0,041 & 0,062 \\
\hline $\mathrm{HCM}$ & 13,26 & 10,39 & 12,31 & 0,032 & 0,061 \\
\hline Hemoglobina & 40,99 & 25,11 & 40,78 & 0,022 & 0,082 \\
\hline CHCM & 3,40 & 1,73 & 2,88 & 0,032 & 0,075 \\
\hline Hematócrito & 34,51 & 20,69 & 33,59 & 0,021 & 0,112 \\
\hline VCM & 24,01 & 22,51 & 22,81 & 0,032 & 0,063 \\
\hline RDW & 27,27 & 21,21 & 25,65 & 0,041 & 0,073 \\
\hline Basófilos & 2,22 & 3,04 & 1,71 & 0,042 & 0,051 \\
\hline Linfócitos & 25,84 & 25,6 & 30,47 & 0,043 & 0,031 \\
\hline Eosinófilos & 23,33 & 21,26 & 21,29 & 0,042 & 0,053 \\
\hline Segmentados & 30,29 & 32 & 30,81 & 0,041 & 0,054 \\
\hline Neutrófilos & 11,93 & 11,26 & 11,05 & 0,042 & 0,041 \\
\hline Plaquetas & 17,7 & 9,96 & 11,6 & 0,031 & 0,033 \\
\hline Bastonetes & 6,82 & 17,65 & 11,76 & 0,022 & 0,044 \\
\hline
\end{tabular}

$\mathrm{NA}=$ não se aplica; ENA = Endocrinologia de adultos; END = Endocrinologia - Diabetes; ETP =Endocrinologia - Tireoide e Paratireoide; $\mathrm{CKMB}=$ creatina fosfoquinase, isoenzima MB; HCM = hemoglobina corpuscular média; $\mathrm{CHCM}=$ concentração da hemoglobina corpuscular média; VCM = volume corpuscular médio; RDW = Red Cell Distribution Width (Amplitude de Distribuição dos Glóbulos Vermelhos) 
Tabela 3. Análise da comparação dos elementos de exames alterados em bioquímica, hormônios, hemograma e urina rotina, realizados nos ambulatórios de Cardiologia e Endocrinologia do Hospital das Clínicas da Faculdade de Medicina de Ribeirão Preto da Universidade de São Paulo (HCFMRP-USP), no período entre julho de 2012 e julho de 2013

\begin{tabular}{|c|c|c|c|}
\hline Ambulatório & ENA \% & END+ETP\% & $\begin{array}{c}\text { Valor de } \mathbf{p} \\
(\text { ENA versus } \text { END+ETP) }\end{array}$ \\
\hline TSH & 45,65 & 49,82 & 0,061 \\
\hline T4 total & 72,73 & 66,67 & 0,042 \\
\hline T4 livre & 36,89 & 29,85 & 0,032 \\
\hline T3 total & 40,40 & 28,87 & 0,022 \\
\hline T3 livre & 57,58 & 39,20 & 0,031 \\
\hline Cálcio iônico & 37,23 & 24,57 & 0,031 \\
\hline Sódio & 13,52 & 14,24 & 0,178 \\
\hline Potássio & 19,32 & 20,63 & 0,181 \\
\hline Proteinúria & 78,13 & 75 & 0,091 \\
\hline Ac TSH (Trab) & 48,65 & 38,39 & 0,042 \\
\hline Hemograma & ENA \% & END+ETP \% & \\
\hline VCM & 22,51 & 22,81 & 0,176 \\
\hline Sementados & 32,00 & 30,81 & 0,189 \\
\hline Neutrófilos & 11,26 & 11,05 & 0,192 \\
\hline Plaquetas & 9,96 & 11,60 & 0,172 \\
\hline PDW & 23,91 & 23,32 & 0,199 \\
\hline PCT & 3,48 & 4,57 & 0,185 \\
\hline MPV & 25,22 & 24,92 & 0,188 \\
\hline Monócitos & 9,98 & 10,92 & 0,212 \\
\hline Linfócitos & 25,60 & 30,47 & 0,168 \\
\hline Eosinófilos & 21,26 & 21,29 & 0,345 \\
\hline Basófilos & 3,04 & 1,71 & 0,061 \\
\hline Bastonetes & 17,65 & 11,76 & 0,056 \\
\hline Hematócrito & 20,69 & 33,59 & 0,051 \\
\hline $\mathrm{HCM}$ & 10,39 & 12,31 & 0,234 \\
\hline Hemoglobina & 25,11 & 40,78 & 0,052 \\
\hline Glóbulos vermelhos & 28,02 & 36,63 & 0,061 \\
\hline Glóbulos brancos & 14,22 & 14,72 & 0,871 \\
\hline $\mathrm{CHCM}$ & 1,73 & 2,88 & 0,222 \\
\hline RDW & 21,21 & 25,65 & 0,234 \\
\hline Urina rotina & ENA \% & END+ETP \% & \\
\hline Ácido ascórbico & 2,70 & 2,56 & 0,877 \\
\hline Bilirrubina & 0,32 & 1,07 & 0,058 \\
\hline Células epiteliais & 54,05 & 53,02 & 0,898 \\
\hline Cilindros & 5,41 & 9,85 & 0,042 \\
\hline Corpos cetônicos & 2,70 & 2,56 & 0,982 \\
\hline Cristais & 14,67 & 16,27 & 0,765 \\
\hline Densidade & 78,38 & 78,40 & 0,987 \\
\hline Glicose & 21,62 & 26,17 & 0,048 \\
\hline Hemácias & 29,73 & 32,26 & 0,256 \\
\hline Heme-pigmento & 8,11 & 8,27 & 0,899 \\
\hline Leucócitos & 33,78 & 33,53 & 0,976 \\
\hline Nitrito & 9,46 & 7,00 & 0,654 \\
\hline $\mathrm{PH}$ & 18,92 & 14,98 & 0,556 \\
\hline Proteínas & 20,27 & 21,98 & 0,875 \\
\hline Urobilinogênio & 16,22 & 10,60 & 0,123 \\
\hline
\end{tabular}

ENA: Endocrinologia de adultos; END: Endocrinologia - Diabetes; ETP: Endocrinologia - Tireoide e Paratireoide; TSH = hormônio estimulante da tireóide; Ac TSH = anticorpo anti-TSH; VCM = volume corpuscular médio; PDW = amplitude de distribuição plaquetária; PCT = plaquetócrito; $\mathrm{MPV}=$ volume plaquetário médio; $\mathrm{HCM}$ = hemoglobina corpuscular média; $\mathrm{CHCM}$ = concentração da hemoglobina corpuscular média; RDW = Red Cell Distribution Width (Amplitude de Distribuição dos Glóbulos Vermelhos); PH = potencial hidrogeniônico 
Tabela 4. Síntese da distribuição das diferenças de resultados dos elementos de exames alterados em Cardiologia e Endocrinologia no Hospital das Clínicas da Faculdade de Medicina de Ribeirão Preto da Universidade de São Paulo (HCFMRP-USP), no período entre julho de 2012 e julho de 2013

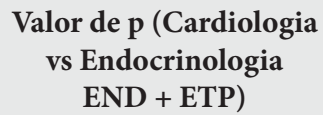

Quantidade de

elementos (\%)

Cardiologia

Endocrinologia END + ETP

Endocrinologia ENA
$11514(20,79)$

$17947(21,85)$

$5691(22,39)$
Valor de $\mathbf{p}$

(Cardiologia vs

Endocrinologia ENA)
Valor de $\mathbf{p}$ (Endocrinologia

ENA vs Endocrinologia END + ETP)

ENA: Endocrinologia de adultos; END: Endocrinologia - Diabetes; ETP: Endocrinologia - Tireoide e Paratireoide

parâmetros anormais, considerando-se que, em muitos casos, os pacientes já foram triados e apresentam um perfil diferente do esperado para um ambulatório de média ou baixa complexidade.

Em Endocrinologia, analisando todos os ambulatórios, o valor médio de alteração dos elementos foi de apenas 22,39\%, muito próximo ao valor encontrado em Cardiologia. Uma particularidade observada nos ambulatórios estudados foi que não houve diferença expressiva na normalidade dos elementos entre os ambulatórios ENA e END + ETP, sendo que, nos exames T4 total e livre, T3 total e livre, cálcio iônico e Ac TSH e em alguns subelementos do hemograma e da urina rotina foram encontradas diferenças estatisticamente significativas $(\mathrm{p}<0,05)$. Tais achados sugerem que, mesmo em ambulatórios em que os pacientes já chegam triados de segunda consulta, como é o caso do ENA, pode haver a possibilidade de solicitação de exames sem necessidade.

$\mathrm{Na}$ Tabela 4, é apresentado um consolidado final sobre os resultados dos exames nos ambulatórios estudados.

Como já foi discutido, os resultados de normalidade para os ambulatórios de Cardiologia e Endocrinologia foram semelhantes, com $79,21 \%$ e $78,15 \%$, respectivamente. Nos ambulatórios estudados, as diferenças encontradas nos valores de exames alterados foram estatisticamente significativas $(p<0,05)$ entre a Cardiologia e a Endocrinologia ENA e END + ETP. No entanto, ao se comparar somente a Endocrinologia em seus dois ambulatórios, não foi verificada diferença significativa $(\mathrm{p}>0,05)$.

Em suma, considerando-se a base de exames estudados, o ambulatório de Cardiologia (CAA) foi onde houve o maior número de exames/paciente observados $(10,36)$, em comparação com o de Endocrinologia (5,91). Tal diferença pode ser explicada em razão do menor número de pacientes nesse último ambulatório e, consequentemente, do menor número de exames analisados, o que é resultante, por conseguinte, de em uma base mais reduzida de pacientes, bem como por causa da diferença de fatores de prevalência e demanda das doenças nos dois ambulatórios estudados.

\section{DISCUSSÃO}

No presente estudo, observou-se que o número de exames por consulta nas duas especialidades (Cardiologia e Endocrinologia) foi de 1,81 e 1,29, respectivamente, o que demonstra que, quando comparada ao padrão sugerido oficialmente, sobretudo em Cardiologia, essa relação é elevada, pois, dentro do recomendável pelo SUS para pacientes ambulatoriais, seria de 0,3 a 0,6 exame $^{14}$.

Quanto à análise qualitativa dos exames laboratoriais e seus elementos abordados neste estudo, os resultados considerados dentro da faixa de normalidade nos ambulatórios de Cardiologia e Endocrinologia foram semelhantes, com 79,21\% e 78,15\%, respectivamente. Esse achado reafirma o que se encontra na literatura, tal como citado por Miyakis et al. ${ }^{15}$ Dessa forma, o valor de normalidade dos elementos, próximo a $80 \%$ nos dois ambulatórios, pode indicar umas das premissas de que, segundo os autores ${ }^{15}$, os exames estão sendo solicitados sem a real e adequada necessidade. Por exemplo, em alguns exames como sódio e potássio, ou em alguns elementos contidos no hemograma (séries vermelha e branca), ou na urina de rotina, os valores de normalidade foram considerados muito altos (acima de $80 \%)$. Isso, de certa forma, pode levantar a questão se, de fato, a solicitação do exame foi adequada e correta segundo os protocolos, como citado por vários autores, ${ }^{15-18}$ os quais defendem o uso de exames complementares como sendo desnecessários quando um resultado normal não foi usado para excluir a suspeita diagnóstica; ou um teste repetido não foi utilizado para a monitorização do tratamento; ou o resultado do teste não fez qualquer diferença para o curso do diagnóstico/tratamento do paciente; ou não ocorreu nenhuma mudança no quadro clínico do paciente que justificasse a solicitação de mais exames. Em outras palavras, a justificativa de solicitação não encontra amparo clínico e científico.

Em razão de a presente pesquisa ter sido realizada em um hospital universitário, ou seja, aquele com finalidades de aprendizado, ensino e pesquisa, além das características assistenciais, é esperado um número mais elevado de exames 
com resultados considerados "normais", por apresentar, em seu corpo médico, estudantes de Medicina ou profissionais com pouco tempo de experiência, ou que ainda estão desenvolvendo e aprimorando as competências médicas/analíticas necessárias, o que difere, por exemplo, do perfil de profissionais de um hospital tradicional dentro da rede atenção. Nesse sentido, está citado na literatura que uma taxa entre 60 e $68 \%$ de exames clínicos laboratoriais poderiam ter sido evitados ou foram realizados sem necessidade ${ }^{15,19}$. Oportunamente, Gortmaker et al. ${ }^{20}$ apontaram que, em situações de pré-operatório, a normalidade de exames laboratoriais fica ainda mais evidente, pois apenas 1,8\% dos exames realizados realmente levou a uma mudança de conduta em relação ao procedimento cirúrgico.

Indubitavelmente, é importante ressaltar que outra variável relevante para o estudo seria relacionada ao perfil e nível do local onde se realiza o atendimento ao paciente, por exemplo, se em enfermaria ou em ambulatório. Assim, estudos ${ }^{20-22}$ mostraram que a frequência média de resultados alterados nas enfermarias foi maior (44\%) que nos ambulatórios (30\%). Tal achado se explica pelo nível de complexidade dos procedimentos realizados e pelo perfil dos pacientes que frequentam o setor de enfermaria em um hospital.

Finalizando, em relação aos resultados de alteração e normalidade obtidos neste estudo, quando separados por área, como Hematologia, Bioquímica e Urinálise, nada há na literatura especializada sobre os resultados esperados dos exames/elementos que foram estudados, dificultando o confrontamento dos resultados aqui apresentados, o que se constitui em uma das limitações do estudo. Além disso, pode-se considerar que, ao não se verificar as condições clínicas dos pacientes, para os quais foram solicitados os exames nos dois ambulatórios, por meio de análise de prontuários ou outros meios, não foi possível discutir em profundidade o contexto e as particularidades possivelmente envolvidas, bem como suas repercussões clínicas, epidemiológicas e econômicas.

Infelizmente, notou-se que, mesmo sendo uma instituição de ensino com respaldo e respeitabilidade por toda a comunidade científica nacional e internacional, a adesão aos protocolos clínicos relacionados à solicitação de exames ainda não é regra. Para se ter uma ideia objetiva dos fatos, somente no ano de $2016^{23}$ aproximadamente $11 \%$ de todos os exames solicitados não tiveram seus resultados nem mesmo checados uma única vez, reforçando a não necessidade e a inadequação quando da sua solicitação.

Diante dos resultados deste estudo, fica evidente, especialmente para profissionais da saúde e, sobretudo, para os gestores da saúde, a importância do tema, muito em sintonia com atual contexto de saúde pública no Brasil. Entender o comportamento de médicos na solicitação dos exames laboratoriais em um hospital de grande porte e referência nacional pode estimular os líderes e os gestores a debater propostas em potencial para melhorar o gerenciamento desses grandes centros de alta complexidade assistencial, buscando a eficiência, a qualidade na assistência e no ensino e a racionalização de uso de recursos na área da saúde.

\section{REFERÊNCIAS}

1. Brasil. Ministério da Saúde. Secretaria-Executiva. Área de Economia da Saúde e Desenvolvimento. Avaliação de tecnologias em saúde: ferramentas para a Gestão do SUS. Brasília: Editora do Ministério da Saúde; 2009.

2. Sullivan R, Peppercorn J, Sikora K, Zalcberg J, Meropol NJ, Amir E, et al. Delivering affordable cancer care in high-income countries. Lancet Oncol. 2011;12(10):933-80. http://dx.doi.org/10.1016/S1470-2045(11)70141-3. PMid:21958503.

3. Biroloni D, Helito AS, organizadores. Análise crítica da prática médica. 3. ed. Rio de Janeiro: Elsevier, 2011.

4. Sociedade Brasileira de Patologia Clínica Medicina Laboratorial. O que é patologia clínica/medicina laboratorial. Rio de Janeiro: SBPC, 2016 [citado em 2016 Ago 5]. Disponível em: http://www.sbpc.org. $\mathrm{br} / ? \mathrm{C}=11$

5. Bulusu S. Rational, cost effective use of investigations. Rising workload and costs in diagnostic departments must be contained. BMJ. 2002;325(7357):222. http://dx.doi.org/10.1136/bmj.325.7357.222/a. PMid:12142322.

6. Issa MRN, Isoni NFC, Soares AM, Fernandes ML. Avaliação pré-anestésica e redução dos custos do preparo pré-operatório. Rev Bras Anestesiol. 2011;61(1):60-71. http://dx.doi.org/10.1016/S0034-7094(11)70007-1. PMid:21334508.

7. Freirea LMD, Sodré FL, Oliveira RA, Castilho LN, Faria EC. Controle de qualidade laboratorial pré-analítico: avaliação de solicitações médicas de bioquímicos no Hospital de Clínicas da Universidade Estadual de Campinas, São Paulo, Brasil. Rev Bras Anal Clin. 2008;40(2):143-5.

8. Hering FR. Auditoria em saúde: avaliação da atenção primária em saúde através da solicitação de exames complementares no município de Gravataí [Tese]. Porto Alegre: Programa de Pós-graduação em Saúde Pública, Universidade Federal do Rio Grande do Sul; 2008.

9. Wu AH. Improving the utilization of clinical laboratory tests. J Eval Clin Pract. 1998;4(3):171-81. http://dx.doi.org/10.1046/j.1365-2753.1998.00001.x. PMid:9744706.

10. Bates DW, Boyle DL, Rittenberg E, Kuperman GJ, Ma’Luf N, Menkin V, et al. What proportion of common diagnostic tests appear redundant? Am J Med. 1998;104(4):361-8. http://dx.doi.org/10.1016/S0002-9343(98)00063-1. PMid:9576410.

11. Branger PJ, Van Oers RJ, Van der Wouden JC, van der Lei J. Laboratory services utilization: a survey of repeat in investigations in ambulatory care. Neth J Med. 1995;47(5):208-13. http://dx.doi.org/10.1016/03002977(95)00100-8. PMid:8544891.

12. Dine CJ, Miller J, Fuld A, Bellini LM, Iwashyna TJ. Educating physiciansin-training about resource utilization and their own outcomes of care in 
the inpatient setting. J Grad Med Educ. 2010;2(2):175-80. http://dx.doi. org/10.4300/JGME-D-10-00021.1. PMid:21975616.

13. Maksoud JG. O uso inadequado dos exames complementares. Rev Pediatr (São Paulo). 1995;17(1):3-4.

14. Rede Interagencial de Informações para a Saúde. Indicadores básicos de saúde no Brasil: conceitos e aplicações. Brasília (DF): Organização PanAmericana de Saúde; 2002

15. Miyakis S, Karamanof G, Liontos M, Mountokalakis TD. Factors contributing to inappropriate ordering of tests in an academic medical department and the effect of an educational feedback strategy. Postgrad Med J. 2006;82(974):823-9. http://dx.doi.org/10.1136/pgmj.2006.049551. PMid:17148707.

16. Branger PJ, Van Oers RJ, Van der Wouden JC, van der Lei J. Laboratory services utilization: a survey of repeat investigations in ambulatory care. Neth J Med. 1995;47(5):208-13. http://dx.doi.org/10.1016/0300-2977(95)00100-8. PMid:8544891.

17. Capilheira MF, Santos IS. Epidemiologia da solicitação de exame complementar em consultas médicas. Rev Saude Publica. 2006;40(2):289-97. http://dx.doi. org/10.1590/S0034-89102006000200015. PMid:16583040.

18. Giordano LA, Giordano MV, Giordano EB, Oliveira e Silva R. Exames préoperatórios nas cirurgias ginecológicas eletivas. Femina. 2009;37(11):619-25.
19. Santos JCM Jr. Avaliação medica: o consumo na medicina e a mercantilização da saúde. Rev Bras Coloproctol. 2006;26(1):70-85. http://dx.doi.org/10.1590/ S0101-98802006000100011.

20. Gortmaker SL, Bickford AF, Mathewson HO, Dumbaugh K, Tirrell PC. A successful experiment to reduce unnecessary laboratory use in a community hospital. Med Care. 1988;26(6):631-42. http://dx.doi.org/10.1097/00005650198806000-00011. PMid:3132579.

21. Janssens PM. Managing the demand for laboratory testing: options and opportunities. Clin Chim Acta. 2010;411(21-22):1596-602. http://dx.doi. org/10.1016/j.cca.2010.07.022. PMid:20659442.

22. Attali M, Barel Y, Somin M, Beilinson N, Shankman M, Ackerman A, et al A cost-effective method for reducing the volume of laboratory tests in a university-associated teaching hospital. Mt Sinai J Med. 2006;73(5):787-94. PMid:17008940.

23. Hospital das Clínicas. Faculdade de Medicina de Ribeirão Preto. Universidade de São Paulo. Relatório de Atividades 2016. Ribeirão Preto: HCRP; 2016 [citado em 2016 Ago 5]. Disponível em: https://site.hcrp.usp.br/wp-content/ uploads/2017/09/RelatoriodeAtividades_HCRP-2016.pdf

Recebido em: Set. 04, 2017 Aprovado em: Abr. 05, 2019 